\title{
La National Library for Health del Reino Unido usa un nuevo motor de búsqueda
}

\author{
Por Robin Murray
}

\begin{abstract}
Resumen: El portal de la National Library of Health del Reino Unido es una realidad desde enero de 2005. El artículo explica sus orígenes y las principales prestaciones que ofrece al personal de la salud, a los bibliotecarios y al público en general.
\end{abstract}

Palabras clave: Biblioteca Nacional de Salud, Portales de búsqueda federada, Consorcios de bibliotecas de salud.

\section{Title: The National Library for Health (UK) uses a new search retrieval system}

Abstracts: The National Library for Health portal has been active since January 2005. The article explains how the portal started and the most important features that it offers to health staff, librarians and patients.

Keywords: National Library Health, Single-search portal, Health library consortia.

EN ENERO DEL 2005, se presentó al público el motor de búsqueda federada en web para el personal sanitario, de administración y pacientes que integra los recursos de información nacionales y locales de las bibliotecas del National Health Service (NHS), el servicio de salud del Reino Unido. Su planificación se había iniciado muchos meses antes.

\section{Antecedentes}

Colin Davies, subdirector de las bibliotecas del North West Health Care inició el proyecto del actual sistema de recuperación de información en abril de 2003, en colaboración con la National Electronic Library for Health (NeLH) y la Library and Knowledge Development Network $(L K D N)$, red para el desarrollo bibliotecario y del conocimiento.

En marzo de 2004, Aidan Halligan, subdirector médico del Departamento de Salud del Reino Unido, solicitó a la junta de directores de la $\mathrm{NeLH}$ que iniciase el desa-
En la primavera de 2005, la South Yorkshire Strategic Health Authority (SYSHA) fue la primera SHA en implementar un entorno de búsqueda única para acceder a los catálogos locales de bibliotecas de salud. En primavera-verano de 2004 se había hecho un estudio de viabilidad para implantar la búsqueda federada (o simultánea) pues, aunque la mayoría de documentos en las 12 bibliotecas de la región estaban en catálogos automatizados, los sistemas de gestión no estaban interconectados.

Gary Amos, director de formación de la SYSHA, explica: "nuestro proyecto local encaja con el trabajo que la National Library for Health $(N L H)$ hizo para crear un sistema de búsqueda única, por lo que tuvo sentido combinar nuestro proyecto con el proyecto nacional. El personal sanitario y los estudiantes tienen acceso $24 / 7$ a la base de datos del conocimiento de Yorkshire".

Esto significa que los 4I.000 trabajadores del National Health Service (NHS) en South Yorkshire, que sirven a I,4 millones de personas (cerca de un $3 \%$ de la población de Inglaterra), podrán encontrar contenido de todos sus catálogos locales, con unos 150.000 títulos. Los usuarios se autentificarán de manera habitual y el sistema reconocerá que el Dr. Smith pertenece al Hospital $X$ y le permitirá acceder a los catálogos de las 12 bibliotecas a través de un portal personalizado.

La conexión del contenido local con el del resto del país se realizará en una segunda fase. El uso de las contraseñas Athens permite al sistema localizar geográficamente a los usuarios y ofrecerles los recursos locales. Desarrollos futuros incluirán personalización del servicio según áreas de interés para los usuarios, como por ejemplo salud mental u ortopedia. 
rrollo de la National Library for Health (NLH) o Biblioteca Nacional para la Salud'.

Uno de los principales éxitos de la $N L H$ fue la implantación del motor de búsqueda único -single search engine (SSE) - en enero de 2005. Forma parte del sitio web global de la $N L H$ y lo puede utilizar tanto el personal del NHS como otros usuarios, e integra recursos de información nacionales y locales de las bibliotecas del NHS.

Para desarrollar la nueva herramienta el NHS solicitó la colaboración a la empresa Fretwell-Downing Informatics $(F D I)$, experta en tecnologías para bibliotecas. Para FDI el desarrollo tuvo más interés que el puramente comercial: "todos somos clientes del NHS".

\section{National Library for Health}

Está constituida por la red de bibliotecas de salud e integra servicios de bibliotecas en local con recursos electrónicos a través de una biblioteca consorciada híbrida que da soporte al cuidado de los pacientes y al desarrollo profesional, e-learning e investigación ${ }^{2}$.

En el Reino Unido los médicos están obligados a seguir el modelo de la medicina basada en la eviden- cia (MBE) cuando hacen diagnósticos o prescripciones. "La práctica de la MBE implica integrar las habilidades clínicas individuales con la mejor evidencia externa disponible sobre la eficacia de los tratamientos".

Se entienden como habilidades clínicas individuales las competencias y el juicio que los médicos adquieren a través de su experiencia y la práctica clínica. El incremento de esta capacidad se refleja de varias maneras, pero específicamente en unos diagnósticos más eficientes y en una identificación más considerada y compasiva con las situaciones, derechos y preferencias de los pacientes cuando deben tomar decisiones sobre su salud.

\section{«La búsqueda única de Zportal facilita el proceso a usuarios principiantes 0 que disponen de poco tiempo"}

Como mejor evidencia clínica externa disponible, entendemos la investigación relevante clínicamente, a menudo desde las ciencias básicas de la medicina, pero específicamente desde la investigación clí-

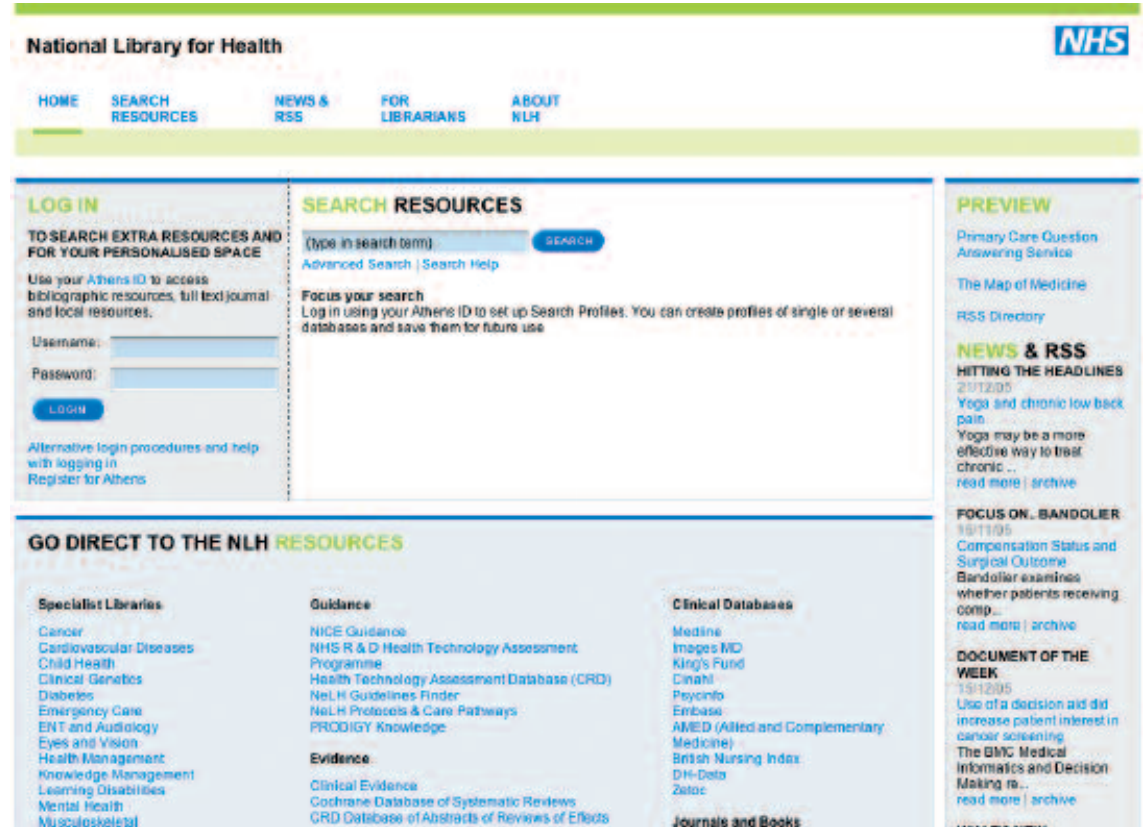

OCLC-PICA adquiere Zportal

En noviembre de 2005 el consorcio mundial de bibliotecas OCLC-PICA adquirió la empresa Fretwell-Downing Informatics (FDI) fabricante, entre otros productos, de Zportal.

DOC6 es distribuidora en España de OCLC desde hace 15 años y de Zportal (FDI) desde marzo de 2005.

nica centrada en el paciente, en la exactitud y la precisión en los tests de diagnósticos (incluyendo el examen clínico) y la eficacia y la seguridad de los regímenes terapéuticos, preventivos y de rehabilitación"”.

\section{Prestaciones y tecnología}

Para lograr el nuevo entorno de búsqueda, el NHS implantó un sistema siguiendo los requerimientos del Official Journal of the European Union y del Managed Services Contract (Mscon). El contrato para desarrollar el motor de búsqueda único se adjudicó a $F D I$ y se basa en la versión 2.7 de Zportal, que ofrece acceso a través de una única consulta a recursos internos y externos. Además integra el gestor de enlaces (open linking, $O L^{2}$ ) junto con otras herramientas de búsqueda propias de FDI.

Aunque estos componentes normalmente se venden de forma conjunta, esta fue la primera vez que $F D I$ tuvo que personalizarlos y reunirlos en un mismo software con funciones adicionales.

Zportal se ha diseñado para integrar el acceso a contenidos diversos, para lo cual utiliza el protocolo Z39.50 junto a Z2Web que incluye motores de búsqueda web.

El contrato para diseñar la interfaz de usuario fue adjudicado a la empresa Oyster, que trabajó con el equipo del $N H S$. 


\section{Años de experiencia}

Esto es lo que EBSCO ofrece.

Nuestro personal gestiona sus suscripciones a revistas electrónicas individuales o incluidas en paquetes de revistas, suscripciones en papel y bases de datoś.

La Lista A-Z (A-to-Z) agrupa todos: sus recursos electrónicos en una misma lista, para que sus usuarios localicen de manera rápida los títulos disponibles y accedan fácilmente a los contenidos. Además, la lista A-to-Z alimenta ál servidor de enlaces LinkSource ${ }^{m}$, que utilizando la norma OpenURL enlaza todos sus recursos de forma compacta e inteligente.

Experiencia, servicio, contenido, soluciones. Hablemos hoy de sus necesidades.

CUSTOMERFOCUSEDCONTENTDRIVEN:

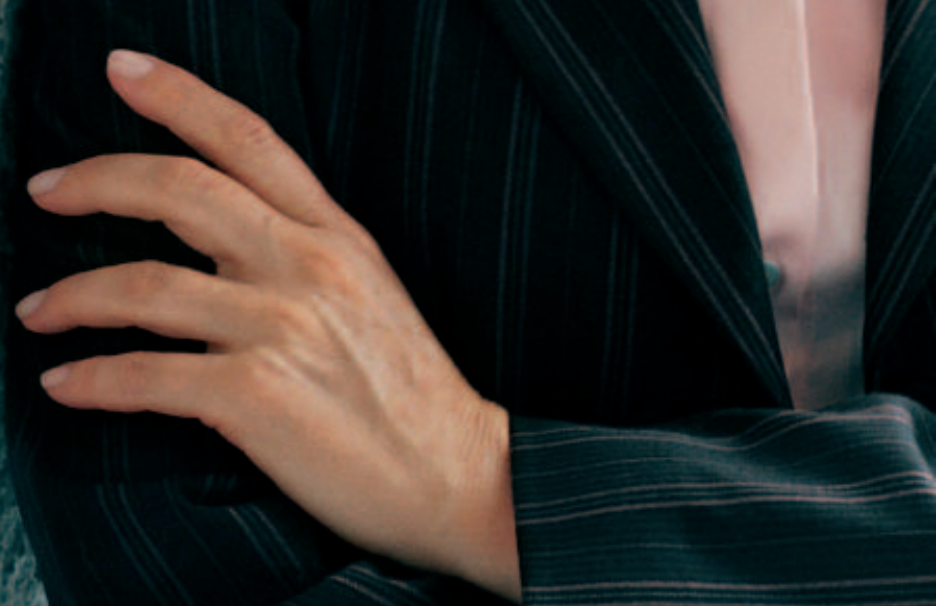
www.ebsco.com
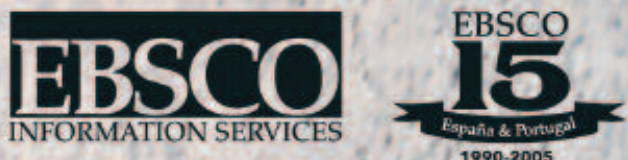


\section{¿Recibes ya EPI en casa?}

Por sólo 75 euros + 4\% IVA puedes tener todos los meses tu copia particular y leerla cómodamente cuando quieras.

Los usuarios anónimos, que no disponen de contraseña, pueden beneficiarse de la información de acceso público. Si se identifican pueden consultar además las suscripciones del NHS. La autentificación y autorización se realiza con $E d u$ serv Athens.

Las interfaces originales de los recursos individuales (por ejemplo, la de Dialog para la consulta de sus bases de datos) siguen estando disponibles para que aquellos que lo deseen no pierdan la posibilidad de búsqueda en la interfaz nativa.

También dispone de una opción de búsqueda avanzada para dar respuesta a las necesidades de usuarios más expertos. Permite interrogar colecciones específicas, usar operadores booleanos y limitar por campos: título, editor, fecha, autor, etc.

Las herramientas de FDI para adaptar las consultas se encuentran en la opción de búsqueda avanzada, funcionan en segundo plano (son transparentes para el usuario) y modifican los criterios de búsqueda según las características de cada recurso. Por ejemplo, NHS Direct sólo permite la consulta por "cualquier" campo. De esta forma se mejora la calidad de los resultados.

Hay aún retos en la búsqueda federada, sobre todo debido a la falta de estándares. No todos los registros tienen el mismo formato; por ejemplo un documento puede estar bajo "Bill Jones", "Jones Bill", "Jones B", etc. Además, todos los sistemas responden a una búsqueda de diferente manera y algunos son más rápidos que otros.

La integración de múltiples recursos requiere la selección de los programas adecuados. Además de Zportal (con Z39.50 y Z2Web) también se incluyen servicios web basados en SOAP, como NeLH. http://www.nelh.nhs.uk

A medida que el proyecto progrese y tenga más contenidos, se ampliará la integración utilizando servicios web basados en SOAP (un protocolo para intercambiar mensajes de xml a través de la Red).

Los sitios se integran de manera personalizada, en función del usuario. Por ejemplo, Zportal puede integrar urls redireccionando al sitio de la fuente cuando el resultado de la búsqueda contenga una url. Es el caso de la mayoría de los resultados de búsqueda en Dialog que incluyen una redirección a la página del sitio web donde está el documento. En otros casos, la solución $O L^{2}$ OpenResolver, de FDI, se utiliza para enlazar al texto completo de proveedores como Ebsco y Biomed Central. Esto significa que los resultados que el usuario recupera desde un agregador (por ejemplo, Dialog) pueden generar un enlace hacia el texto completo del artículo proporcionado por otro proveedor del NHS. Este nivel de integración brinda cada vez más formas de acceso al texto completo de los documentos.

Los resultados se presentan de forma innovadora: bajo unas etiquetas diseñadas por Oyster. FDI previamente ha "mapeado" los resultados de manera que se colocan en diferentes conjuntos definidos por el NHS como: "todos los resultados", "guías", "medicina basada en la evidencia", "bases de datos clínicas", "revistas y libros" y "para los pacientes". La primera pestaña contiene todos los resultados. Las otras se dividen acordes a los criterios del NHS y han requerido algo más de programación.

\section{Segunda fase}

La primera etapa va consolidándose poco a poco. El NHS ha encargado a una empresa externa que analice cómo utilizan el portal los usuarios: movimientos que realizan con el ratón, navegación por el sitio, etc., y qué aspectos pueden ser mejorados. El sistema está configurado para que gestione como mínimo unas 600.000 búsquedas por mes, aunque esta cifra puede ampliarse.

El portal se ha diseñado para poder integrar las iniciativas existentes en tecnologías de la información del NHS incluyendo las del Programa Nacional para la Tecnología de la Información (NPfIT) conocido como Connecting for health, desde el 1 de abril de 2005.

En la segunda fase se introducirán algunas mejoras como un tesauro, más precisión en la localización geográfica, opciones de búsqueda, y más contenidos.

Una etapa futura de la $N L H$ puede ser la integración del SSE con otras aplicaciones que usan los profesionales de la medicina, como por ejemplo documentos de archivo electrónicos, de manera que un médico de cabecera pueda encontrar folletos de información para el paciente o evidencia clínica desde su aplicación de gestión de documentos de archivo electrónicos.

\section{Notas}

1. Consultado en: 24-07-05.

http://www.library.nhs.uk/forlibrarians/

2. De las notas de la reunión plenaria de la NHS library and knowledge development network celebrada el 12 de julio de 2004 en Londres.

3. Sackett, D. L. "Evidence-based medicine". En: Seminars in perinatology, 1997, February, 21, n. 1, pp. 3-5.

4. National Programme for Information Technology en el NHS en Inglaterra.

Robin Murray, managing director, Fretwell-Downing Informatics. http://www.fdisolutions.com robin.murray@fdisolutions.com

Traducido por: Marta Estruch, Recursos Electrónicos Doc6.

mestruch@doc6.es

http://www.doc6.es 